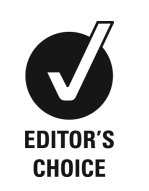

Department of Obstetrics B Maternidade Bissaya BarretoCentro Hospitalar e Universitário de Coimbra, Coimbra, Portugal ${ }^{2}$ Department of Neurology, Centro Hospitalar Universitário de Coimbra, Coimbra, Portugal

\section{Correspondence to} Dr Ana Raquel Neves, anaraquel.Ineves@gmail.com

Accepted 18 December 2014

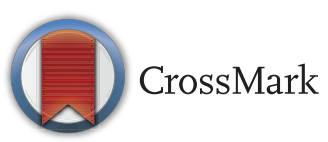

To cite: Neves $A R$, Monteiro P, Matos A, et al. BMJ Case Rep Published online: [please include Day Month Year] doi:10.1136/ bcr-2014-207708

\title{
Anti-MuSK-positive myasthenia gravis diagnosed during pregnancy: new challenges for an old disease?
}

\author{
Ana Raquel Neves, ${ }^{1}$ Pitorra Monteiro, ${ }^{1}$ Anabela Matos, ${ }^{2}$ Isabel Santos Silva ${ }^{1}$
}

\section{SUMMARY}

Myasthenia gravis is an autoimmune disorder affecting predominantly women in their reproductive age. The course of the disease during pregnancy is unpredictable, although it is more difficult to manage earlier in the gestation. Myasthenia gravis with antibodies against the musclespecific receptor tyrosine kinase (anti-MuSK) has been described as a subtype of disease with more localised clinical features and a poorer response to treatment than acetylcholine receptor antibody (anti-AChR)-positive patients. Few cases have been reported in pregnant women, with deliveries being performed mainly by caesarean section. We report a successful case of vaginal delivery and describe our experience providing the first review of the management of this subtype of disease during pregnancy.

\section{BACKGROUND}

Acetylcholine receptor antibody (anti-AChR) antibodies are detected in $75-80 \%$ of patients with generalised myasthenia gravis (MG). Among patients without anti-AChR antibodies, antimusclespecific receptor tyrosine kinase (anti-MuSK) antibodies are detected in $40 \%$ of cases. ${ }^{1}$

Anti-MuSK-positive MG has a marked female preponderance. When comparing to anti-AChR-positive MG the age of onset is in average 10 years later, in the fourth decade. ${ }^{2-5}$ Although bulbar symptoms have been reported to be more common in this subtype of disease, three phenotypes have recently been described: oculopharyngeal weakness, with occasional profound tongue and facial atrophy; neck, shoulder and respiratory weakness without ocular weakness; and a phenotype indistinguishable from anti-AChR-positive MG. Another striking feature of anti-MuSK-positive MG is the poorer response to medical treatment, with a lower rate of complete remission. $^{6-8}$

Since these antibodies were described for the first time, there have been multiple cases reported in literature allowing for a better understanding of the clinical features and appropriate management of these patients. However, few cases of anti-MuSK-positive MG during pregnancy have been described. We report the diagnosis and management of the disease in a pregnant woman, highlighting the importance of a multidisciplinary approach of these patients.

\section{CASE PRESENTATION}

A 39-year-old patient, gravida 3, para 1, with irrelevant medical or familial history, was diagnosed with generalised immune-mediated MG with oculobulbar predominance during the first trimester of pregnancy.

The patient referred a 6-month history of bilateral ptosis, horizontal diplopia and dysphagia. On examination she presented bilateral ptosis, bilateral limitation of eye abduction, diplopia in all directions of gaze, dysphonia, facial diparesis, limited cervical flexion and bilateral superior limb proximal fatigable weakness. There was no evidence of respiratory compromise. Neurophysiological examination with repetitive nerve stimulation of radial, accessory and facial nerves was normal. Thoracic CT showed residual thymic tissue in the anterior mediastinum. Radioimmunoprecipitation assay revealed negative anti-AChR antibodies and positive anti-MuSK antibodies.

After clinical diagnosis of MG the patient initiated treatment with pyridostigmine $(60 \mathrm{mg}$ per os four times a day) with partial benefit on bulbar weakness. At 15 and 19 weeks of pregnancy, she presented two episodes of worsening of myasthenic symptoms, namely dysphagia, diplopia, cervical weakness and ptosis, requiring hospitalisation. Intravenous immunoglobulin (IvIG) $0.4 \mathrm{~g} / \mathrm{kg}$ body weight per day, 5 days per month was introduced. The symptoms improved but did not resolve. After the second episode of exacerbation, $125 \mathrm{mg}$ intravenous methylprednisolone was associated during hospitalisation. Immediately following the corticoid administration, the patient developed an anaphylactic reaction which resolved after intravenous treatment with clemastine. Corticosteroid therapy was suspended and IvIG administration was increased to $0.4 \mathrm{~g} / \mathrm{kg}$ body weight per day, 3 days every 3 weeks. There was no repercussion in the fetal well-being in none of the episodes. Owing to the patient's poor response to treatment, desensitisation therapy was performed at 21 weeks of pregnancy and methylprednisolone was resumed at a dosage of $64 \mathrm{mg}$ per os per day. Medication was maintained until delivery.

Obstetric surveillance was performed according to the hospital protocol, with normal laboratory and ultrasound scan routines. Third trimester ultrasound scan showed a fetus growing in the 25 th centile, with normal amniotic fluid and no signs of fetal akinesia.

The patient presented to the emergency room at 34 weeks and 4 days with preterm premature rupture of membranes (PPROM). On admission amniotic fluid was clear, Bishop score was 5 and cardiotocography was reassuring, demonstrating irregular 
contractility. The patient presented a discrete bilateral ptosis and facial diparesis. Intravenous ampicillin protocol for PPROM was initiated. On re-evaluation $5 \mathrm{~h} 30 \mathrm{~min}$ after admission, Bishop score was 7 and the patient maintained a reassuring cardiotocogra-

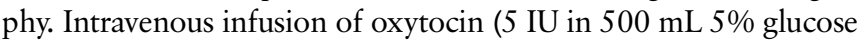
solution) was initiated to regularise uterine contractility. After discussion of the case with the neurology team it was decided that, given the patient's clinical stability, there was no contraindication to vaginal delivery. Ambulatory medication was maintained until delivery. With the beginning of the active phase of labour, intravenous hydrocortisone protocol for prophylaxis of adrenal insufficiency was initiated and, after anaesthetic evaluation, epidural anaesthesia was performed. During labour there was no exacerbation of myasthenic symptoms.

Eutocic delivery was performed uneventfully at $12 \mathrm{~h} 30 \mathrm{~min}$ after rupture of membranes. A lively male infant (weight: 2360 g; APGAR score 9/10/10) was born, showing no signs of muscular weakness.

\section{OUTCOME AND FOLLOW-UP}

Both the mother and the newborn went from the delivery room to the puerperium ward. Breastfeeding was initiated in the first hour postpartum, according to the hospital protocol. The newborn presented no signs of neonatal disease, neither initially nor in the course of time. The mother showed no immediate signs of worsening of myasthenic symptoms. Prepartum MG medication was maintained. Postpartum care was performed according to the hospital protocol and both the mother and the newborn were discharged on the second day.

Three months after, the patient presents only with mild facial diparesis; she is now with prednisolone $20 \mathrm{mg}$ daily per os, in a reducing dosage schedule, and monthly IvIG $(0.4 \mathrm{~g} / \mathrm{kg}$ body weight).

\section{DISCUSSION}

The management of MG during pregnancy is controversial, and so is the mode of delivery.

The few cases reported make it impossible to conclude if the evolution of anti-MuSK-positive MG during pregnancy differs from that in anti-AChR-positive patients. It is known that the course of the disease varies widely between women and even between pregnancies in the same woman. ${ }^{9-12}$ Exacerbations are more likely in the first trimester and after delivery, though there are no predictive risk factors to identify mothers at risk of exacerbation. ${ }^{11}$ The second and third trimesters are usually associated with a remission of symptoms due to the normal immunosuppressive changes occurring during this period. ${ }^{5}{ }^{6}$ In this case, both exacerbations occurred during the second trimester, underlining the importance of a regular evaluation of these patients, both by a neurologist and an obstetrician. It is also consensual that the risk of maternal mortality is highest in the first year of disease. Thus, women should delay pregnancy until the disease is stable. ${ }^{12-15}$ This patient was diagnosed during the first trimester of pregnancy, when the risk is highest.

Although MG does not seem to be associated with an increased risk of spontaneous abortion or preterm labour, Niks et $a l^{16}$ described their experience with an anti-MuSK-positive myasthenic pregnant patient who presented two early spontaneous abortions and questioned a pathogenic effect of anti-MuSK antibodies in utero. 5614
The physiopathological mechanisms by which anti-MuSK antibodies cause myasthenic symptoms are not completely

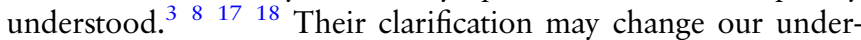
standing of labour in these patients. In the few cases reported, delivery was performed mainly by caesarean section. ${ }^{19-21}$ However, in light of the knowledge currently available, vaginal delivery should be the preferred route for birth in all patients with MG and caesarean section should be performed only for obstetric indications. Since striated muscle is involved in the second stage of labour, assisted delivery is frequently indicated in these patients. ${ }^{5} 111215$ In this case, given the patient's clinical stability and the fact that the third trimester ultrasound showed a fetus in the 25 th centile, we performed an eutocic delivery, with all the advantages for both mother and infant.

The management of the disease during pregnancy is also controversial. In this case, due to the unknown positivity to anti-MuSK antibodies by the time of diagnosis, we initiated therapy with acetylcholinesterase inhibitors and titrated the dose according to the patient's side effects. The response to treatment was poor, which is consistent with this subtype of disease. ${ }^{7223}$ The choice between plasmapheresis and IvIG during pregnancy is also questionable. Kanzaki et $a l^{20}$ described their experience with the use of plasmapheresis in a pregnant woman whose infant developed neonatal MG. Although both plasmapheresis and IvIG seem to be effective in the treatment of myasthenic exacerbations in anti-MuSK-positive MG, plasmapheresis seems to be associated with an increased risk for prematurity. Moreover, due to the physiological changes of coagulation factors that occur during pregnancy, plasmapheresis should be considered a second choice in the treatment of these patients. 571224

Most patients improve their symptoms with steroids or other immunosuppressive drugs. ${ }^{2} 47$ In this case, oral methylprednisolone was initiated after desensitisation due to the persistence of symptoms despite regular therapy with IvIG. With this association, clinical stability was achieved during pregnancy, labour and puerperium. However, it should be noted that the use of methylprednisolone is associated with an increased risk of palate cleft when used in the first trimester and that high doses have been associated with an increased risk of premature rupture of membranes (PRM), as stated in the case above. ${ }^{12} 25$ Irrespective of the treatment with corticosteroids, pregnant patients with MG have a three times higher risk of PRM. It has been suggested that this complication might be a consequence of polyhydramnios associated with neonatal MG. ${ }^{5} 11$ Since in this case the infant showed no prenatal or postnatal signs of disease, we cannot exclude the relation between PRM and the use of corticosteroids during the second and third trimesters.

Other immunosuppressive agents were not considered due to their reported unsafety during pregnancy. ${ }^{5} 12 \quad 14 \quad 26$ Although azathioprine has been considered safe, an increased risk of gestational complications such as prematurity, fetal growth restriction, low birth weight and increased risk of fetal myelosuppression have been described. ${ }^{24}{ }^{26}$ Given the patient's moderate symptoms, we decided not to introduce this agent during pregnancy.

The role of thymectomy in non-thymomatous patients is unclear and it seems to be of little value in anti-MuSK-positive patients. ${ }^{6722}$ Besides, when considered in pregnant patients, it should be performed after a stable postpartum period due to the delayed therapeutic effect and surgical risks. ${ }^{12}$ Therefore, it was not considered in this case. 


\section{Learning points}

- The effect of antimuscle-specific receptor tyrosine kinase (anti-MuSK) antibodies on the mechanism of labour is yet to be clarified.

- Positivity to anti-MuSK antibodies poses an extra challenge in the management of myasthenic patients due to their poorer response to the classical therapeutic approaches.

- Eutocic delivery should be the first option in clinically stable pregnant women with myasthenia gravis and caesarean section should be performed only for obstetric indications.

- Since the course of disease during pregnancy is unpredictable, a multidisciplinary approach is essential in the management of these patients.

Acknowledgements The authors wish to thank Dr Ana Rita Borges and Dr José Coelho for their contribution in data collection.

Contributors ARN was involved in the conception, designing, data collection and drafting of the article. PM contributed to the drafting and revision of the obstetrical clinical aspects of the case. AM contribted to the drafting and revision of the neurological clinical aspects of the case. ISS contributed both to the drafting of the case and to the final revision and approval of the version published.

Competing interests None.

Patient consent Obtained.

Provenance and peer review Not commissioned; externally peer reviewed.

\section{REFERENCES}

1 Eymard B. Antibodies in myasthenia gravis. Rev Neurol 2009;165:137-43.

2 Evoli A, Tonali PA, Padua L, et al. Clinical correlates with anti-MuSK antibodies in generalized seronegative myasthenia gravis. Brain 2003;126(Pt 10):2304-11.

3 Vincent A, McConville J, Farrugia M, et al. Seronegative myasthenia gravis. Semin Neurol 2004;24:125-33.

4 Guptill JT, Sanders DB, Evoli A. Anti-musk antibody myasthenia gravis: clinical findings and response to treatment in two large cohorts. Muscle Nerve 2011;44:36-40.

5 Varner M. Myasthenia gravis and pregnancy. Clin Obstet Gynecol 2013;56:372-81.
6 Statland JM, Ciafaloni E. Myasthenia gravis: five new things. Neurol Clin Pract 2013:3:126-33

7 Evoli $A$, Bianchi MR, Riso $R$, et al. Response to therapy in myasthenia gravis with anti-MuSK antibodies. Ann N Y Acad Sci 2008;1132:76-83.

8 Baggi F, Andreetta F, Maggi L, et al. Complete stable remission and autoantibody specificity in myasthenia gravis. Neurology 2013;80:188-95.

9 Djelmis J, Sostarko M, Mayer D, et al. Myasthenia gravis in pregnancy: report on 69 cases. Eur J Obs Gynecol Reprod Biol 2002;104:21-5.

10 Ferrero $\mathrm{S}$, Esposito F, Biamonti M, et al. Myasthenia gravis during pregnancy. Expert Rev Neurother 2008;8:979-88.

11 Téllez-Zenteno JF, Hernández-Ronquillo L, Salinas V, et al. Myasthenia gravis and pregnancy: clinical implications and neonatal outcome. BMC Musculoskelet Disord 2004;5:42.

12 Massey JM, De Jesus-Acosta C. Pregnancy and myasthenia gravis. Continuum (Minneap Minn) 2014;20(1 Neurology of Pregnancy):115-27.

13 Ramirez C, de Seze J, Delrieu O, et al. Myasthenia gravis and pregnancy: clinical course and management of delivery and the postpartum phase. Rev Neurol 2006;162:330-8.

14 Chaudhry SA, Vignarajah B, Koren G. Motherisk update myasthenia gravis during pregnancy. Can Fam Physician 2012;58:1346-9.

15 Norwood F, Dhanjal M, Hill M, et al. Myasthenia in pregnancy: best practice guidelines from a UK multispecialty working group. J Neurol Neurosurg Psychiatry 2014;85:538-43.

16 Niks EH, Verrips A, Semmekrot BA, et al. A transient neonatal myasthenic syndrome with anti-MuSK antibodies. Neurology 2008;70:1215-16.

17 Vincent A, Bowen J, Newsom-Davis J, et al. Seronegative generalised myasthenia gravis: clinical features, antibodies, and their targets. Lancet Neurol 2003;2:99-106.

18 McConville J, Farrugia M, Beeson D, et al. Detection and characterization of MuSK antibodies in seronegative myasthenia gravis. Ann Neurol 2004;55:580-4.

19 Terrero A, Ramírez-Rivera J. "Seronegative" anti-MUSK positive myasthenia gravis presenting during pregnancy. Bol Asoc Med P R 2006;98:210-12.

20 Kanzaki A, Motomura M. A pregnant patient with anti-MuSK antibody positive myasthenia gravis and her infant with transient neonatal myasthenia gravis. Rinsho Shinkeigaku 2011;51:188-91.

21 Béhin A, Mayer M, Kassis-Makhoul B, et al. Severe neonatal myasthenia due to maternal anti-MuSK antibodies. Neuromuscul Disord 2008;18:443-6.

22 Oh SJ. Muscle-specific receptor tyrosine kinase antibody positive myasthenia gravis current status. J Clin Neurol 2009;5:53-64.

23 Gilhus NE, Owe JF, Hoff JM, et al. Myasthenia gravis: a review of available treatment approaches. Autoimmune Dis 2011;2011:847393.

24 Berlit S, Tuschy B, Spaich S, et al. Myasthenia gravis in pregnancy: a case report. Case Rep Obstet Gynecol 2012;2012:736024.

25 Schneider-Gold C, Gajdos P, Toyka K, et al. Corticosteroids for myasthenia gravis. Cochrane Database Syst Rev 2005;(2):CD002828.

26 Motta M, Tincani A, Meroni $P$, et al. Follow-up of children exposed antenatally to immunosuppressive drugs. Rheumatology (Oxford) 2008;47(Suppl 3):32-4.

Copyright 2015 BMJ Publishing Group. All rights reserved. For permission to reuse any of this content visit

http://group.bmj.com/group/rights-licensing/permissions.

BMJ Case Report Fellows may re-use this article for personal use and teaching without any further permission.

Become a Fellow of BMJ Case Reports today and you can:

- Submit as many cases as you like

- Enjoy fast sympathetic peer review and rapid publication of accepted articles

- Access all the published articles

- Re-use any of the published material for personal use and teaching without further permission

For information on Institutional Fellowships contact consortiasales@bmjgroup.com

Visit casereports.bmj.com for more articles like this and to become a Fellow 